\title{
Crystal structure of bis(4,4,5,5-tetramethyl-2-(thiophenal-2-yl)- imidazoline-1-oxyl-3-oxide)-bis(hexafluoroacetylacetonato)nickel(II), $\mathrm{Ni}\left(\mathrm{C}_{11} \mathrm{H}_{15} \mathrm{~N}_{2} \mathrm{O}_{2} \mathrm{~S}\right)_{2}\left[\mathrm{C}_{3} \mathrm{HO}_{2}\left(\mathrm{CF}_{3}\right)_{2}\right]_{2}$
}

\author{
Xiao-Song Wang', Xia Gao', Xiao-Lei Hou ${ }^{*}$,II and Xiao-Qiang Wang ${ }^{*}$,II \\ I Henan Institute of Education, Department of Chemistry, Zhengzhou 450046, P. R. China \\ II Henan Provincial Nonferrous Metals Geological Exploration, Total Institute, Zhengzhou 450052, P. R. China
}

Received May 6, 2009, accepted and available on-line June 8, 2009; CCDC no. 1267/2649

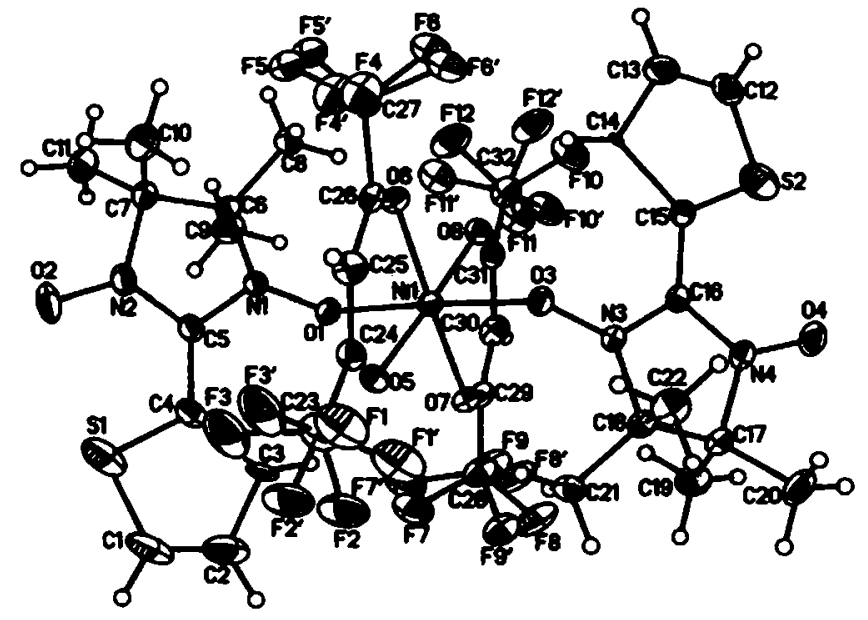

Abstract

$\mathrm{C}_{32} \mathrm{H}_{32} \mathrm{~F}_{12} \mathrm{~N}_{4} \mathrm{NiO}_{8} \mathrm{~S}_{2}$, monoclinic, $P 121 / c 1$ (no. 14), $a=12.1886(9) \AA, b=15.137(1) \AA, c=22.367(2) \AA$, $\beta=101.895(1)^{\circ}, V=4038.1 \AA^{3}, Z=4, R_{g r}(F)=0.073$, $w R_{\text {ref }}\left(F^{2}\right)=0.239, T=291 \mathrm{~K}$.

\section{Source of material}

NIT2-thp was prepared according to a literature method [1]. The title complex was synthesized as follows: a mixture of $\mathrm{Ni}(\mathrm{hfac})_{2}$. $2 \mathrm{H}_{2} \mathrm{O}$ (77 mg, $0.15 \mathrm{mmol}$ ) and NIT2-thp ( $36 \mathrm{mg}, 0.15 \mathrm{mmol}$ ) was dissolved with heating to $75^{\circ} \mathrm{C}$ in heptane/acetone $(10 \mathrm{ml} / 10 \mathrm{ml})$ mixture, the resulting solution was stirred for $1 \mathrm{~h}$ and then cooled to room temperature. After filtration, the filtrate was allowed to stand at room temperature for $72 \mathrm{~h}$ to produce well-shaped darkgreen crystals. Elemental analysis - found: $\mathrm{C}, \mathbf{4 0 . 3 2 \%} \mathrm{H}$, $3.36 \%$; $\mathrm{N}, 5.91 \%$; calculated for $\mathrm{C}_{32} \mathrm{H}_{32} \mathrm{~F}_{12} \mathrm{~N}_{4} \mathrm{NiO}_{8} \mathrm{~S}_{2}$ : C, $40.39 \% ; H, 3.39 \% ; \mathrm{N}, 5.89 \%$.

\section{Experimental details}

The $\mathrm{CF}_{3}$-groups were handled according to the disorder in the crystal structure, which caused larger residual values.

\section{Discussion}

During the past decade, nitronyl nitroxide radicals (NITR) have been widely employed as molecular units in the design and synthesis of molecular magnetic materials [2-4]. The nitroxide derivatives can be bound to the metal through the oxygen atom of $\mathrm{O}-\mathrm{N}$ groups, resulting in a variety of transition metal-radical complexes have [5-9]. Although the family of nitronyl nitroxides has

been widely studied for a long time, the NITR $(R=$ methyl, ethyl, phenyl, pyridyl etc.), thiophenalyl substituents have rarely been reported so far. Therefore, in order to extend our knowledge of the extremely rich chemistry of such systems, we are devoted to the explorations of new building block for molecule-based magnetic materials. In this paper, we have synthesized a novel nickel(II) complex involving thiophene-substituted nitronyl nitroxide radical (NIT2-thp).

In the title crystal structure the nickel atom is hexa-coordinated in a distorted octahedral environment. The apical positions are occupied by two oxygen atoms ( $\mathrm{O} 1$ and $\mathrm{O} 3$ ) of the nitronyl nitroxide radicals, and the equatorial positions are occupied by four oxygen atoms $(\mathrm{O} 5,06,07,08)$ of two hfac molecules. The $\mathrm{Ni} 1-\mathrm{O} 1$ and $\mathrm{Nil}-\mathrm{O} 3$ bond length (2.105(3) $\AA$ and 2.099(3) $\AA$, respectively are larger than the $\mathrm{Nil}-\mathrm{O} 5, \mathrm{Nil}-\mathrm{O6}, \mathrm{Ni}-\mathrm{O} 7$ and $\mathrm{Nil}-\mathrm{O} 8$ bond lengths (2.021(3) $\AA, 2.007(3) \AA, 2.011(3) \AA$ and 2.023(3) $\AA$, respectively). The coordinated $\mathrm{N}-\mathrm{O}$ bond lengths of $\mathrm{N} 1-\mathrm{Ol}$ and $\mathrm{N} 3-\mathrm{O} 3$ are $1.284(5) \AA$ and 1.295(5) $\AA$, respectively, which is similar to the values observed in nitroxide (NIT2-thp, the $\mathrm{N}-\mathrm{O}$ bond lengths are 1.285(5) $\AA$ and 1.282(5) $\AA$ ), which indicates the nitroxide oxygen atoms are only very loosely bonded to the central $\mathrm{Ni}(\mathrm{II})$ ion in the apical direction. The uncoordinated $\mathrm{N}-\mathrm{O}$ bond lengths of $\mathrm{N} 2-\mathrm{O} 2$ and $\mathrm{N} 4-\mathrm{O} 4$ are 1.267(6) $\AA$ and $1.285(6) \AA$, respectively. Bond distances and angles in the hfac molecules are in the normal range for this kind of compound [1012]. The dihedral angle between the thiophene ring $(C 1, C 2, C 3$, $\mathrm{C} 4, \mathrm{~S} 1)$ and the mean plane of the nitroxide radical $(\mathrm{O} 2, \mathrm{~N} 2, \mathrm{C} 5$, $\mathrm{N} 1, \mathrm{O} 1)$ is $15.9^{\circ}$. From the unit cell packing, two adjacent molecules of the title complex are linked by pairs of inversion-related $0 \cdots S$ weak intermolecular interactions involving $04 \mathrm{~B} \cdots \mathrm{S} 2 \mathrm{~A}$ and S2B $\cdots$ O4A, resulting in a dimeric structure, the distance of $\mathrm{O} \cdots \mathrm{S}$ is $3.118 \AA$.

Table 1. Data collection and handling.

\section{Crystal:}

Wavelength:

$\mu$ :

Diffractometer, scan mode: $2 \theta_{\max }$ :

$N(h k l)_{\text {measured, }} N(h k l)_{\text {unique: }}$ Criterion for $I_{\mathrm{obs}}, N(\mathrm{hkl})_{\mathrm{gt}}$ :

$N(\text { param })_{\text {refined : }}$

Programs: dark-green block, size $0.29 \times 0.34 \times 0.45 \mathrm{~mm}$ Mo $K_{\alpha}$ radiation $(0.71073 \AA)$ $6.91 \mathrm{~cm}^{-1}$

Bruker SMART CCD, $\varphi / \omega$ $51^{\circ}$

21447,7472

$I_{\mathrm{obs}}>2 \sigma\left(I_{\mathrm{obs}}\right), 5698$

580

SHELXS-97 [13], SHELXL-97 [14], SHELXTL [15]

\footnotetext{
* Correspondence author (e-mail: flywangxq@ 163.com)
} 
Table 2. Atomic coordinates and displacement parameters (in $\AA^{2}$ ).

\begin{tabular}{llllll}
\hline Atom & Site & $x$ & $y$ & $z$ & $U_{\text {iso }}$ \\
\hline $\mathrm{H}(1)$ & $4 e$ & -0.1550 & -0.0072 & 0.9126 & 0.127 \\
$\mathrm{H}(2)$ & $4 e$ & -0.1504 & -0.0024 & 0.8110 & 0.128 \\
$\mathrm{H}(3)$ & $4 e$ & -0.0096 & 0.1085 & 0.7798 & 0.064 \\
$\mathrm{H}(8 \mathrm{~A})$ & $4 e$ & 0.4098 & 0.2463 & 0.8734 & 0.089 \\
$\mathrm{H}(8 \mathrm{~B})$ & $4 e$ & 0.4123 & 0.3498 & 0.8718 & 0.089 \\
$\mathrm{H}(8 \mathrm{C})$ & $4 e$ & 0.3610 & 0.2967 & 0.8125 & 0.089 \\
$\mathrm{H}(9 \mathrm{~A})$ & $4 e$ & 0.1798 & 0.3671 & 0.7953 & 0.104 \\
$\mathrm{H}(9 \mathrm{~B})$ & $4 e$ & 0.2226 & 0.4313 & 0.8502 & 0.104 \\
$\mathrm{H}(9 \mathrm{C})$ & $4 e$ & 0.1129 & 0.3761 & 0.8478 & 0.104 \\
$\mathrm{H}(10 \mathrm{~A})$ & $4 e$ & 0.3509 & 0.2427 & 1.0209 & 0.135 \\
$\mathrm{H}(10 \mathrm{~B})$ & $4 e$ & 0.4270 & 0.2782 & 0.9776 & 0.135 \\
$\mathrm{H}(10 \mathrm{C})$ & $4 e$ & 0.3607 & 0.1895 & 0.9621 & 0.135 \\
$\mathrm{H}(11 \mathrm{~A})$ & $4 e$ & 0.1820 & 0.4191 & 0.9470 & 0.134 \\
$\mathrm{H}(11 \mathrm{~B})$ & $4 e$ & 0.3119 & 0.4229 & 0.9721 & 0.134 \\
$\mathrm{H}(11 \mathrm{C})$ & $4 e$ & 0.2336 & 0.3802 & 1.0116 & 0.134 \\
$\mathrm{H}(12)$ & $4 e$ & 0.6821 & 0.1385 & 0.6021 & 0.086 \\
\hline
\end{tabular}

Table 2. Continued.

\begin{tabular}{llllll}
\hline Atom & Site & $x$ & $y$ & $z$ & $U_{\text {iso }}$ \\
\hline H(13) & $4 e$ & 0.6991 & 0.1395 & 0.7062 & 0.095 \\
H(14) & $4 e$ & 0.5452 & 0.0513 & 0.7491 & 0.024 \\
H(19A) & $4 e$ & 0.1454 & -0.0263 & 0.5833 & 0.115 \\
H(19B) & $4 e$ & 0.0769 & -0.1129 & 0.5636 & 0.115 \\
H(19C) & $4 e$ & 0.1504 & -0.0716 & 0.5209 & 0.115 \\
H(20A) & $4 e$ & 0.2486 & -0.2176 & 0.5212 & 0.127 \\
H(20B) & $4 e$ & 0.1793 & -0.2581 & 0.5663 & 0.127 \\
H(20C) & $4 e$ & 0.3107 & -0.2590 & 0.5832 & 0.127 \\
H(21A) & $4 e$ & 0.1695 & -0.1368 & 0.7324 & 0.100 \\
H(21B) & $4 e$ & 0.1064 & -0.1853 & 0.6733 & 0.100 \\
H(21C) & $4 e$ & 0.1135 & -0.0819 & 0.6747 & 0.100 \\
H(22A) & $4 e$ & 0.4032 & -0.2166 & 0.6784 & 0.106 \\
H(22B) & $4 e$ & 0.2964 & -0.2705 & 0.6847 & 0.106 \\
H(22C) & $4 e$ & 0.3541 & -0.2076 & 0.7376 & 0.106 \\
H(25) & $4 e$ & 0.4010 & -0.0756 & 0.9221 & 0.067 \\
H(30) & $4 e$ & 0.1407 & 0.2455 & 0.6168 & 0.068 \\
\hline
\end{tabular}

Table 3. Atomic coordinates and displacement parameters (in $\AA^{2}$ ).

\begin{tabular}{|c|c|c|c|c|c|c|c|c|c|c|c|}
\hline Atom & Site & Occ. & $x$ & $y$ & $z$ & $U_{11}$ & $U_{22}$ & $U_{33}$ & $U_{12}$ & $U_{13}$ & $U_{23}$ \\
\hline$F(1)$ & $4 e$ & $0.670(6)$ & $0.2510(6)$ & $-0.1931(4)$ & $0.9041(4)$ & $0.105(4)$ & $0.059(3)$ & $0.151(5)$ & $0.003(3)$ & $0.044(4)$ & $0.046(4)$ \\
\hline$F(2)$ & $4 e$ & 0.670 & $0.1003(6)$ & $-0.1437(6)$ & $0.8481(3)$ & $0.093(4)$ & $0.102(5)$ & $0.119(6)$ & $-0.042(3)$ & $0.016(4)$ & $0.030(4)$ \\
\hline$F(3)$ & $4 e$ & 0.670 & $0.1607(8)$ & $-0.0942(6)$ & $0.9363(4)$ & $0.138(6)$ & $0.134(6)$ & $0.132(5)$ & $-0.020(4)$ & $0.081(5)$ & $0.029(4)$ \\
\hline$F(4)$ & $4 e$ & $0.61(3)$ & $0.595(1)$ & $-0.0308(6)$ & $0.9447(6)$ & $0.075(4)$ & $0.073(3)$ & $0.071(4)$ & $0.026(3)$ & $-0.008(3)$ & $0.009(3)$ \\
\hline$F(5)$ & $4 e$ & 0.61 & $0.557(1)$ & $0.1071(7)$ & $0.9532(6)$ & $0.063(4)$ & $0.064(3)$ & $0.060(5)$ & $-0.013(3)$ & $0.001(3)$ & $0.000(3)$ \\
\hline$F(6)$ & $4 e$ & 0.61 & $0.628(1)$ & $0.063(1)$ & $0.8780(5)$ & $0.050(2)$ & $0.094(6)$ & $0.095(3)$ & $0.000(4)$ & $0.015(2)$ & $0.004(4)$ \\
\hline$F(7)$ & $4 e$ & $0.69(1)$ & $-0.0885(8)$ & $0.1346(9)$ & $0.6643(4)$ & $0.057(3)$ & $0.131(6)$ & $0.118(4)$ & $-0.003(4)$ & $0.013(3)$ & $0.015(4)$ \\
\hline$F(8)$ & $4 e$ & 0.69 & $-0.0341(7)$ & $0.0562(5)$ & $0.5960(6)$ & $0.096(4)$ & $0.079(3)$ & $0.099(5)$ & $-0.002(3)$ & $-0.040(4)$ & $-0.021(3)$ \\
\hline$F(9)$ & $4 e$ & 0.69 & $-0.0442(6)$ & $0.1969(5)$ & $0.5870(3)$ & $0.069(3)$ & $0.063(3)$ & $0.074(3)$ & $0.010(3)$ & $-0.020(3)$ & $0.001(3)$ \\
\hline$F(10)$ & $4 e$ & $0.75(1)$ & $0.4340(6)$ & $0.2709(5)$ & $0.6395(4)$ & $0.090(4)$ & $0.106(4)$ & $0.100(5)$ & $-0.014(3)$ & $0.043(3)$ & $0.027(3)$ \\
\hline$F(11)$ & $4 e$ & 0.75 & $0.2850(5)$ & $0.3476(4)$ & $0.6181(3)$ & $0.093(4)$ & $0.065(3)$ & $0.088(3)$ & $0.001(3)$ & $0.025(3)$ & $0.034(3)$ \\
\hline$F(12)$ & $4 e$ & 0.75 & $0.3883(9)$ & $0.3490(5)$ & $0.7086(3)$ & $0.147(6)$ & $0.091(4)$ & $0.081(4)$ & $-0.061(4)$ & $0.011(3)$ & $-0.001(3)$ \\
\hline$F\left(1^{\prime}\right)$ & $4 e$ & 0.33 & $0.210(1)$ & $-0.1934(7)$ & $0.8526(6)$ & $0.105(4)$ & $0.059(3)$ & $0.151(5)$ & $0.003(3)$ & $0.044(4)$ & $0.046(4)$ \\
\hline$F\left(2^{\prime}\right)$ & $4 e$ & 0.33 & $0.0861(7)$ & $-0.112(1)$ & $0.8769(7)$ & $0.093(4)$ & $0.102(5)$ & $0.119(6)$ & $-0.042(3)$ & $0.016(4)$ & $0.030(4)$ \\
\hline$F\left(3^{\prime}\right)$ & $4 e$ & 0.33 & $0.231(1)$ & $-0.147(1)$ & $0.9423(4)$ & $0.138(6)$ & $0.134(6)$ & $0.132(5)$ & $-0.020(4)$ & $0.081(5)$ & $0.029(4)$ \\
\hline$F\left(4^{\prime}\right)$ & $4 e$ & 0.39 & $0.582(2)$ & $-0.019(1)$ & $0.9570(8)$ & $0.075(4)$ & $0.073(3)$ & $0.071(4)$ & $0.026(3)$ & $-0.008(3)$ & $0.009(3)$ \\
\hline$F\left(5^{\prime}\right)$ & $4 e$ & 0.39 & $0.573(2)$ & $0.1212(7)$ & $0.9381(9)$ & $0.063(4)$ & $0.064(3)$ & $0.060(5)$ & $-0.013(3)$ & $0.001(3)$ & $0.000(3)$ \\
\hline$F\left(6^{\prime}\right)$ & $4 e$ & 0.39 & $0.623(2)$ & $0.031(2)$ & $0.8737(8)$ & $0.050(2)$ & $0.094(6)$ & $0.095(3)$ & $0.000(4)$ & $0.015(2)$ & $0.004(4)$ \\
\hline$F\left(7^{\prime}\right)$ & $4 e$ & $0.31(2)$ & $-0.072(2)$ & $0.172(1)$ & $0.6655(8)$ & $0.057(3)$ & $0.131(6)$ & $0.118(4)$ & $-0.003(4)$ & $0.013(3)$ & $0.015(4)$ \\
\hline$F\left(8^{\prime}\right)$ & $4 e$ & 0.31 & $-0.046(2)$ & $0.171(1)$ & $0.5742(5)$ & $0.096(4)$ & $0.079(3)$ & $0.099(5)$ & $-0.002(3)$ & $-0.040(4)$ & $-0.021(3)$ \\
\hline $\mathbf{F}\left(9^{\prime}\right)$ & $4 e$ & 0.31 & $-0.050(1)$ & $0.0489(5)$ & $0.6234(9)$ & $0.069(3)$ & $0.063(3)$ & $0.074(3)$ & $0.010(3)$ & $-0.020(3)$ & $0.001(3)$ \\
\hline$F\left(10^{\prime}\right)$ & $4 e$ & $0.25(1)$ & $0.314(2)$ & $0.330(2)$ & $0.6049(4)$ & $0.090(4)$ & $0.106(4)$ & $0.100(5)$ & $-0.014(3)$ & $0.043(3)$ & $0.027(3)$ \\
\hline$F\left(11^{\prime}\right)$ & $4 e$ & 0.25 & $0.322(2)$ & $0.3647(8)$ & $0.6974(7)$ & $0.093(4)$ & $0.065(3)$ & $0.088(3)$ & $0.001(3)$ & $0.025(3)$ & $0.034(3)$ \\
\hline$F\left(12^{\prime}\right)$ & $4 e$ & 0.25 & $0.4558(8)$ & $0.293(1)$ & $0.673(1)$ & $0.147(6)$ & $0.091(4)$ & $0.081(4)$ & $-0.061(4)$ & $0.011(3)$ & $-0.001(3)$ \\
\hline $\mathrm{Ni}(1)$ & $4 e$ & & $0.27005(5)$ & $0.08209(4)$ & $0.76829(3)$ & $0.0386(4)$ & $0.0380(4)$ & $0.0319(4)$ & $-0.0047(2)$ & $0.0064(2)$ & $0.0013(2)$ \\
\hline$S(1)$ & $4 e$ & & $-0.0170(2)$ & $0.0928(2)$ & $0.9422(1)$ & $0.094(2)$ & $0.091(2)$ & $0.154(2)$ & $-0.006(1)$ & $0.069(2)$ & $0.021(2)$ \\
\hline$S(2)$ & $4 e$ & & $0.5322(2)$ & $0.0494(2)$ & $0.5820(1)$ & $0.086(1)$ & $0.088(1)$ & $0.111(2)$ & $-0.001(1)$ & $0.036(1)$ & $0.019(1)$ \\
\hline$O(1)$ & $4 e$ & & $0.1813(3)$ & $0.1865(2)$ & $0.7986(2)$ & $0.046(2)$ & $0.043(2)$ & $0.042(2)$ & $0.000(2)$ & $0.007(2)$ & $-0.006(2)$ \\
\hline$O(2)$ & $4 e$ & & $0.1120(5)$ & $0.2400(4)$ & $0.9895(2)$ & $0.122(5)$ & $0.126(5)$ & $0.076(3)$ & $-0.029(4)$ & $0.068(3)$ & $-0.028(3)$ \\
\hline$O(3)$ & $4 e$ & & $0.3518(3)$ & $-0.0243(2)$ & $0.7360(2)$ & $0.047(2)$ & $0.049(2)$ & $0.038(2)$ & $0.004(2)$ & $0.006(2)$ & $-0.006(2)$ \\
\hline$O(4)$ & $4 e$ & & $0.3814(4)$ & $-0.0839(3)$ & $0.5378(2)$ & $0.092(3)$ & $0.079(3)$ & $0.043(2)$ & $-0.013(2)$ & $0.025(2)$ & $-0.014(2)$ \\
\hline$O(5)$ & $4 e$ & & $0.2038(3)$ & $-0.0072(2)$ & $0.8180(2)$ & $0.046(2)$ & $0.045(2)$ & $0.049(2)$ & $-0.004(2)$ & $0.015(2)$ & $0.006(2)$ \\
\hline$O(6)$ & $4 e$ & & $0.4081(3)$ & $0.0877(2)$ & $0.8349(2)$ & $0.046(2)$ & $0.042(2)$ & $0.040(2)$ & $-0.005(2)$ & $0.003(2)$ & $0.002(1)$ \\
\hline$O(7)$ & $4 e$ & & $0.1339(3)$ & $0.0774(2)$ & $0.7000(2)$ & $0.047(2)$ & $0.042(2)$ & $0.044(2)$ & $-0.005(2)$ & $-0.001(2)$ & $-0.001(2)$ \\
\hline$O(8)$ & $4 e$ & & $0.3373(3)$ & $0.1747(2)$ & $0.7214(2)$ & $0.047(2)$ & $0.048(2)$ & $0.041(2)$ & $-0.002(2)$ & $0.011(2)$ & $0.008(2)$ \\
\hline$N(1)$ & $4 e$ & & $0.1911(3)$ & $0.2175(3)$ & $0.8529(2)$ & $0.042(2)$ & $0.039(2)$ & $0.041(2)$ & $-0.002(2)$ & $0.013(2)$ & $-0.002(2)$ \\
\hline$N(2)$ & $4 e$ & & $0.1569(5)$ & $0.2442(3)$ & $0.9433(2)$ & $0.074(3)$ & $0.062(3)$ & $0.059(3)$ & $-0.009(3)$ & $0.033(3)$ & $-0.011(2)$ \\
\hline$N(3)$ & $4 e$ & & $0.3309(3)$ & $-0.0572(3)$ & $0.6814(2)$ & $0.042(2)$ & $0.039(2)$ & $0.039(2)$ & $-0.001(2)$ & $0.009(2)$ & $-0.004(2)$ \\
\hline $\mathrm{N}(4)$ & $4 e$ & & $0.3460(4)$ & $-0.0864(3)$ & $0.5881(2)$ & $0.058(3)$ & $0.046(2)$ & $0.039(2)$ & $-0.002(2)$ & $0.011(2)$ & $-0.006(2)$ \\
\hline$C(1)$ & $4 e$ & & $-0.1055(7)$ & $0.0294(6)$ & $0.8977(6)$ & $0.062(5)$ & $0.086(6)$ & $0.18(1)$ & $-0.018(4)$ & $0.058(6)$ & $0.012(6)$ \\
\hline $\mathrm{C}(2)$ & $4 e$ & & $-0.1030(8)$ & $0.0329(6)$ & $0.8392(6)$ & $0.077(5)$ & $0.075(5)$ & $0.17(1)$ & $-0.027(4)$ & $0.034(6)$ & $-0.015(6)$ \\
\hline$C(3)$ & $4 e$ & & $-0.0214(4)$ & $0.0955(3)$ & $0.8186(3)$ & $0.029(2)$ & $0.039(3)$ & $0.099(5)$ & $-0.016(2)$ & $0.029(3)$ & $-0.016(3)$ \\
\hline$C(4)$ & $4 e$ & & $0.0363(5)$ & $0.1311(4)$ & $0.8819(3)$ & $0.051(3)$ & $0.047(3)$ & $0.086(4)$ & $0.000(2)$ & $0.033(3)$ & $0.000(3)$ \\
\hline $\mathrm{C}(5)$ & $4 e$ & & $0.1265(4)$ & $0.1946(3)$ & $0.8919(2)$ & $0.050(3)$ & $0.045(3)$ & $0.054(3)$ & $-0.002(2)$ & $0.021(2)$ & $-0.003(2)$ \\
\hline$C(6)$ & $4 e$ & & $0.2555(4)$ & $0.3011(3)$ & $0.8732(2)$ & $0.049(3)$ & $0.038(3)$ & $0.049(3)$ & $-0.007(2)$ & $0.013(2)$ & $-0.007(2)$ \\
\hline$C(7)$ & $4 e$ & & $0.2581(5)$ & $0.2994(4)$ & $0.9421(3)$ & $0.062(3)$ & $0.061(3)$ & $0.050(3)$ & $-0.010(3)$ & $0.018(3)$ & $-0.013(3)$ \\
\hline$C(8)$ & $4 e$ & & $0.3703(5)$ & $0.2982(4)$ & $0.8561(3)$ & $0.057(3)$ & $0.059(3)$ & $0.067(4)$ & $-0.020(3)$ & $0.023(3)$ & $-0.016(3)$ \\
\hline
\end{tabular}


Table 3. Continued.

\begin{tabular}{|c|c|c|c|c|c|c|c|c|c|c|c|}
\hline Atom & Site & Occ. & $x$ & $y$ & $z$ & $U_{11}$ & $U_{22}$ & $U_{33}$ & $U_{12}$ & $U_{13}$ & $U_{23}$ \\
\hline$C(9)$ & $4 e$ & & $0.1863(6)$ & $0.3758(4)$ & $0.8384(3)$ & $0.078(4)$ & $0.047(3)$ & $0.082(4)$ & $-0.003(3)$ & $0.011(3)$ & $0.001(3)$ \\
\hline$C(10)$ & $4 e$ & & $0.3587(7)$ & $0.2475(6)$ & $0.9792(3)$ & $0.088(5)$ & $0.128(7)$ & $0.050(4)$ & $-0.004(5)$ & $0.003(4)$ & $0.002(4)$ \\
\hline$C(11)$ & $4 e$ & & $0.2452(7)$ & $0.3886(5)$ & $0.9709(4)$ & $0.102(6)$ & $0.083(5)$ & $0.089(5)$ & $-0.018(4)$ & $0.034(5)$ & $-0.043(4)$ \\
\hline$C(12)$ & $4 e$ & & $0.6325(6)$ & $0.1074(5)$ & $0.6208(4)$ & $0.069(4)$ & $0.065(4)$ & $0.093(5)$ & $-0.017(3)$ & $0.046(4)$ & $-0.004(4)$ \\
\hline$C(13)$ & $4 e$ & & $0.6412(6)$ & $0.1087(5)$ & $0.6813(4)$ & $0.063(4)$ & $0.065(4)$ & $0.113(6)$ & $-0.018(3)$ & $0.023(4)$ & $-0.023(4)$ \\
\hline$C(14)$ & $4 e$ & & $0.5540(3)$ & $0.0585(2)$ & $0.7090(2)$ & $0.012(2)$ & $0.023(2)$ & $0.029(2)$ & $-0.009(1)$ & $0.015(1)$ & $-0.011(1)$ \\
\hline$C(15)$ & $4 e$ & & $0.4814(4)$ & $0.0216(3)$ & $0.6433(2)$ & $0.045(3)$ & $0.037(2)$ & $0.049(3)$ & $0.001(2)$ & $0.011(2)$ & $-0.006(2)$ \\
\hline$C(16)$ & $4 e$ & & $0.3877(4)$ & $-0.0368(3)$ & $0.6383(2)$ & $0.045(3)$ & $0.034(2)$ & $0.039(2)$ & $0.003(2)$ & $0.008(2)$ & $-0.004(2)$ \\
\hline$C(17)$ & $4 e$ & & $0.2452(5)$ & $-0.1371(4)$ & $0.5943(3)$ & $0.053(3)$ & $0.048(3)$ & $0.052(3)$ & $-0.007(2)$ & $0.008(2)$ & $-0.014(2)$ \\
\hline$C(18)$ & $4 e$ & & $0.2614(5)$ & $-0.1397(3)$ & $0.6649(3)$ & $0.052(3)$ & $0.038(3)$ & $0.056(3)$ & $-0.008(2)$ & $0.013(2)$ & $-0.007(2)$ \\
\hline$C(19)$ & $4 e$ & & $0.1452(6)$ & $-0.0818(5)$ & $0.5626(3)$ & $0.062(4)$ & $0.093(5)$ & $0.066(4)$ & $-0.003(4)$ & $-0.008(3)$ & $-0.003(4)$ \\
\hline$C(20)$ & $4 e$ & & $0.2460(8)$ & $-0.2261(5)$ & $0.5634(4)$ & $0.115(6)$ & $0.056(4)$ & $0.087(5)$ & $-0.025(4)$ & $0.030(5)$ & $-0.032(4)$ \\
\hline$C(21)$ & $4 e$ & & $0.1526(5)$ & $-0.1355(4)$ & $0.6885(3)$ & $0.058(4)$ & $0.062(4)$ & $0.085(4)$ & $-0.022(3)$ & $0.029(3)$ & $-0.016(3)$ \\
\hline$C(22)$ & $4 e$ & & $0.3356(6)$ & $-0.2156(4)$ & $0.6941(3)$ & $0.084(5)$ & $0.051(3)$ & $0.076(4)$ & $0.000(3)$ & $0.013(4)$ & $0.013(3)$ \\
\hline$C(23)$ & $4 e$ & & $0.1941(5)$ & $-0.1222(4)$ & $0.8856(3)$ & $0.086(5)$ & $0.063(4)$ & $0.079(5)$ & $-0.007(4)$ & $0.026(4)$ & $0.029(4)$ \\
\hline$C(24)$ & $4 e$ & & $0.2588(5)$ & $-0.0487(3)$ & $0.8620(2)$ & $0.058(3)$ & $0.040(3)$ & $0.049(3)$ & $-0.003(2)$ & $0.019(3)$ & $0.006(2)$ \\
\hline$C(25)$ & $4 e$ & & $0.3693(5)$ & $-0.0370(4)$ & $0.8910(3)$ & $0.064(4)$ & $0.051(3)$ & $0.051(3)$ & $0.000(3)$ & $0.005(3)$ & $0.016(3)$ \\
\hline$C(26)$ & $4 e$ & & $0.4339(4)$ & $0.0302(3)$ & $0.8754(2)$ & $0.051(3)$ & $0.043(3)$ & $0.032(2)$ & $0.003(2)$ & $0.005(2)$ & $-0.002(2)$ \\
\hline$C(27)$ & $4 e$ & & $0.5548(5)$ & $0.0415(3)$ & $0.9127(2)$ & $0.049(3)$ & $0.061(4)$ & $0.060(3)$ & $0.010(3)$ & $0.005(3)$ & $0.004(3)$ \\
\hline$C(28)$ & $4 e$ & & $-0.0154(6)$ & $0.1316(3)$ & $0.6276(2)$ & $0.068(4)$ & $0.058(4)$ & $0.069(4)$ & $0.010(3)$ & $-0.016(3)$ & $-0.001(3)$ \\
\hline$C(29)$ & $4 e$ & & $0.1070(4)$ & $0.1376(3)$ & $0.6615(2)$ & $0.050(3)$ & $0.045(3)$ & $0.041(3)$ & $0.003(2)$ & $0.000(2)$ & $-0.004(2)$ \\
\hline$C(30)$ & $4 e$ & & $0.1716(5)$ & $0.2065(4)$ & $0.6478(2)$ & $0.071(4)$ & $0.051(3)$ & $0.041(3)$ & $0.004(3)$ & $-0.002(3)$ & $0.010(2)$ \\
\hline$C(31)$ & $4 e$ & & $0.2820(5)$ & $0.2194(3)$ & $0.6791(2)$ & $0.060(3)$ & $0.045(3)$ & $0.038(3)$ & $-0.003(2)$ & $0.017(2)$ & $0.000(2)$ \\
\hline$C(32)$ & $4 e$ & & $0.3454(5)$ & $0.2986(4)$ & $0.6615(2)$ & $0.091(5)$ & $0.058(4)$ & $0.048(3)$ & $-0.012(3)$ & $0.014(3)$ & $0.011(3)$ \\
\hline
\end{tabular}

Acknowledgments. This work was supported by the National Natural Science Foundation of China (grant no. 20471026) and the Natural Science Foundation of Henan province (grant no. 0311021200 ).

\section{References}

1. Ullman, E. F.; Call, L.; Osieckei, J. H.: Stable free radicals. VIII. New imino, amidino, and carbamoyl nitroxides. J. Org. Chem. 35 (1970) 36233628.

2. Luneau, D.; Romero, F. M.; Ziessel, R.: Nitronyl Nitroxide Biradicals as Tetradentate Chelates: Unusually Large Metal-Nitroxide Ferromagnetic Interactions. Inorg. Chem. 37 (1998) 5078-5087.

3. Deumal, M.; Cirujeda, J.; Veciana, J.; Novoa, J. J.: Structure-Magnetism Relationships in Nitronyl Nitroxide Radicals. Chem. Eur. J. 5 (1999) 1631-1642.

4. Sporer, C.; Ruiz-Molina, D.; Wurst, K.; Kopacka, H.; Veciana, J.; Jaitner, $P$.: Ferrocene substituted nitronyl nitroxide and imino nitroxide radicals. Synthesis, X-ray structure and magnetic properties. J. Organomet. Chem. 507 (2001) 637-639.

5. Li, L. C.; Liao, D. Z.; Liu, S. Y.; Jiang, Z. H.; Yan, S. P.: A onedimensional chain compound [Cu(im2-py)(SCN)2 $]_{n}$ exhibiting strong ferromagnetic coupling. Inorg. Chem. Commun. 6 (2003) 225-228.

6. Wang, L. Y.; Liu, Z. L.; Liao, D. Z.; Jiang, Z. H.; Yan, S. P.: Synthesis, structure and magnetic behavior of a novel 1-D chain tethered by nickel(II)-4-pyridyl-substituted nitronyl nitroxide radical complex and TCB. Inorg. Chem.Commun. 6 (2003) 630-633.

7. Mathevet, F.; Lunean, D.: Interpenetrated 3D Polymeric Metal-Radical Networks Built from a Tetranitroxide Radical and Bis(hexafluoroacethylacetonato)Manganese(II). J. Am. Chem. Soc. 123 (2001) 7465 7466.

8. Ogita, M.; Yamamoto, Y.; Suzuki, T.; Kaizaki, S.: Syntheses, Structures, and Spectroscopic Properties of Cobalt(III) Complexes Containing 3- or 4-Pyridyl-Substituted Nitronyl and Imino Nitroxide Ligands. Eur. J. Inorg. Chem. 4 (2002) 886-894.
9. Yamamoto, Y.; Yoshida T.; Suzuki, T; Kaizaki, S.: Crystal structures of cobalt(II), nickel(II) and zinc(II) dichloro complexes bearing 2-pyridylsubstituted nitronyl nitroxide (NIT2py). Inorg. Chim. Acta. 325 (2001) 187-192.

10. Wang, H. M.; Liu, Z. L.; Liu, C. M.; Zhang, D. Q.; Lu, Z. L.; Geng, H.; Shuai, Z. G.; Zhu, D. B.: Coordination Complexes of 2-(4-Quinolyl) nitronyl Nitroxide with $\mathrm{M}(\mathrm{hfac})_{2}[\mathrm{M}=\mathrm{Mn}$ (II), $\mathrm{Co}$ (II), and $\mathrm{Cu}(\mathrm{II})]$ : Syntheses, Crystal Structures, and Magnetic Characterization. Inorg. Chem. 43 (2004) 4091-4098.

11. Smith, C. D.; Bottle, S. E.; Junk, P. C.; Inoue, K.; Markosyan, A. S.: Synthesis and properties of $\mathrm{Mn}(\mathrm{hfac})_{2}$ complexes of isoindoline nitroxide radicals. Synthetic Metals 138 (2003) 501-506.

12. Rajadurai, C.; Falk, K.; Ostrovsky, S.; Enkelmann, V.; Haase, W. Baumgarten, $\mathrm{M}$.: 1-D coordination chains of $\mathrm{Cu}(\mathrm{hfac})_{2}$ and $\mathrm{Mn}(\mathrm{hfac})_{2}$ bridged by phenylnitronylnitroxide derivatives. Inorg. Chim. Acta 358 (2005) 3391-3397.

13. Sheldrick, G. M.: SHELXXS-97. Program for the Solution of Crystal Structures. University of Göttingen, Germany 1997.

14. Sheldrick, G. M.: SHELXL-97. Program for the Refinement of Crystal Structures. University of Gōttingen, Germany 1997.

15. Sheldrick, G. M.: SHELXTL. Structure Determination Software Suite. Version 6.14. Bruker AXS, Madison, Wisconsin, USA 2000. 